\title{
Joint Paediatric-Orthodontic Course: A team approach to children's dental care
}

The annual 'Joint Paediatric-Orthodontic Course' took place on Friday 31 January 2020 at etc.venues in London. The course was collaboratively organised by the Royal College of Surgeons Faculty of Dental Surgery, British Orthodontic Society (BOS) and British Society of Paediatric Dentistry (BSPD). Following an excellent spread of continental breakfast, Dr Jayne Owen, Specialist in Paediatric Dentistry and Chair of BSPD Specialist Branch, gave us all a very warm welcome, highlighting key topics in which both paediatric dentists and orthodontists work together to achieve better outcomes for patients. Topics such as dental trauma, molarincisor hypomineralisation and cleft lip/ palate featured prominently.

This was followed by Dr Mariyah Nazir, Consultant Orthodontist at Manchester Dental Hospital, presenting us with an engaging talk on the orthodontic perspective of managing hypodontia patients. This included challenging case studies where functional considerations, aesthetics and the impact of restorative burden were explored in depth. The cases showed that having complex interdisciplinary management whilst maintaining patient autonomy allowed for successful outcomes. Dr Frankie Soldani, Consultant in Paediatric Dentistry at Community Dental Services in Bradford, followed with a talk on managing patients who suffer from Severe Combined Immunodeficiency (SCID) and the associated dental and orthodontic implications. The importance of including carers and other healthcare workers in treatment planning was emphasised. A thought-provoking case study showed us the work of a creative team using valpast flexible overdentures to bring back a patient's function and smile. The morning concluded with Dr Simon Littlewood, Consultant Orthodontist at St Luke's Hospital, Bradford exploring the challenges faced when managing patients with Amelogenesis Imperfecta. Important information regarding improving bonding of orthodontic brackets and the benefits of temporary anchorage devices was given.

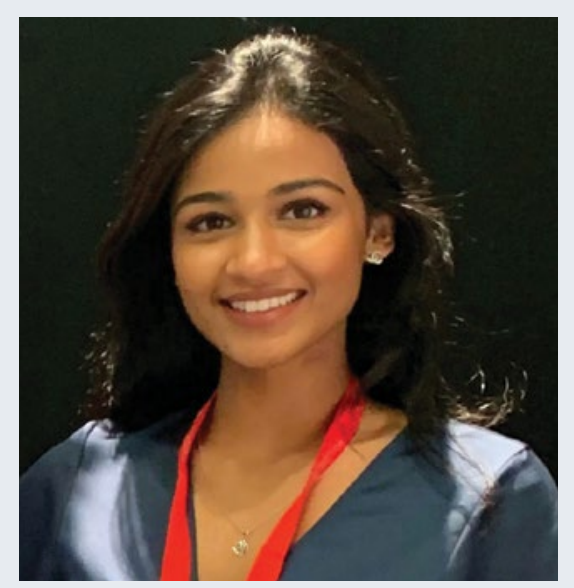

In the afternoon, Dr Soldani graced us for a second time giving an update on trauma injuries in patients prior to and during orthodontic treatment. She also went on to educate us on how trauma can be managed with orthodontics. The fundamentals of auto-transplantation, decoronation and apexification were presented thoroughly, with much audience participation. The course was brought to a close by Dr Stephen Chadwick, Consultant Orthodontist at Countess of Chester Hospital, captivating us with his enjoyable and informative talk on interceptive orthodontics and when to step in with paediatric cases. Contemporary concepts such as space maintainers and retained deciduous dentition were discussed, alongside fascinating case studies and examples.

Overall, the course was extremely insightful and rewarding, a view echoed by the majority of attendees. Knowledge was disseminated effectively in a packed schedule that ran its course seamlessly. I would like to thank all the speakers for their inspiring lectures and especially $\mathrm{Dr}$ Jayne Owen and Dr Simon Littlewood as the co-convenors for an excellently organised course. My knowledge and confidence in the topics concerned has been greatly enhanced. I look forward to attending the course again next year and would encourage all colleagues with an interest in children's dental care to attend in future.

By Sanjana Sudarshan, DCT2 Paediatric Dentistry,

Royal London Hospital, Barts Health NHS Trust 